\title{
Acyclic subgraph is hard
}

\author{
Thinh D. Nguyen \\ Moscow State University \\ kosmofarmer@gmail.com
}

\begin{abstract}
We prove the hardness of yet another problem in graph theory, namely ACYCLIC SUBGRAPH. A reduction from INDEPENDENT SET shows that our claim holds.
\end{abstract}

Keywords: acyclic, edge, graph, independent, vertex

\section{Introduction}

In this section, we give the description of the problem and necessary notions from graph theory.

\subsection{GRAPH THEORY NOTIONS}

A graph $G=(V, E)$ is an undirected graph with the vertex set $V=V(G)$ and edge set $E=E(G)$. In a graph, every edge $(u, v)(u, v \in V)$ is undirected. Digraph is a different notion, a digraph $D=(V, A)$ is a directed graph with the vertex set $V=V(D)$ and arc set $A=A(D)$. In a digraph, every $\operatorname{arc}(u, v)(u, v \in V)$ is directed, meaning that $u$ is the starting vertex of the arc, and $v$ is the ending vertex of the arc. A cycle in a digraph $D$ is a sequence of vertices $\left(v_{1}, v_{2}, \ldots, v_{k}\right)$ in which, for all $1 \leq i<k,\left(v_{i}, v_{i+1}\right)$ is an $\operatorname{arc}$ of $D$, and also $\left(v_{k}, v_{1}\right)$ is an arc of $D$. A digraph without any cycle is called a $D A G$ (Directed Acyclic Graph). In an (undirected) graph, two vertices $u, v$ are adjacent if $(u, v)$ is an edge.

In a graph $G$, a set $V^{\prime} \subset V$ is an independent set if $V^{\prime}$ has no pair of adjacent vertices. A well-known result states that the problem of finding the largest independent set InDEPENDENT SET is hard.

Definition 1.1. Independent $\operatorname{Set}=\left\{\langle G, k\rangle \mid\right.$ the graph $G$ has an independent set $V^{\prime} \subset V(G)$ containing $k$ vertices\}

\subsection{ACYCLIC SUBGRAPH}

Now, we are going to give the definitions that are pertinent to our soon-to-be-defined problem. The notion of induced subgraph of an undirected graph is well studied. But, in this article, we consider the notion of induced subgraph of a digraph. The concept is similar. An induced subgraph of a digraph $D=(V, A)$ is also determined by its vertex set $V^{\prime} \subset V$. Its arc set is all the arc in $V^{\prime} \times V^{\prime} \cap A(D)$. Our problem is to find the largest DAG subgraph of a given digraph.

Definition 1.2. ACYClic SUbGraph $=\left\{\langle D, k>|\right.$ the digraph $D$ has an induced DAG subgraph on $V^{\prime} \subset V(D)$ containing $k$ vertices, in other words, $D\left[V^{\prime}\right]$ is a DAG and $\left.\left|V^{\prime}\right|=k\right\}$

We also utilize the notation of the induced subgraph by $V^{\prime} \subset V$ in a digraph $D=(V, A)$ by $D\left[V^{\prime}\right]$.

\section{Main claim and proof}

Claim 2.1. Independent SET $\leq_{p}$ ACYClic Subgraph

Proof. For an instance of Independent Set $G=(V, E)$ and an integer $k$, create a digraph $D=(V, A)$ as follows. The vertex set $V$ is the same as in $G$. For each edge $(u, v) \in E(G)$, we creat two arcs $(u, v)$ and $(v, u)$ in $A(D)$. Clearly, if $G$ has an independent set $V^{\prime}$ of size $k$, the induced subgraph of $V^{\prime}$ in $D$ is necessarily a DAG. Since a cycle in $D\left[V^{\prime}\right]$ would have some arc, and an arc in $D$ corresponds to an edge in $G$. Conversely, if $D$ has a induced DAG subgraph $D\left[V^{\prime}\right]$ with $\left|V^{\prime}\right|=k$ then $V^{\prime}$ must form an independent set in $G$. Assume to the contrary that $\left|V^{\prime}\right|$ contains an edge $(u, v)$ in $G$, that edge necessarily corresponds to two $\operatorname{arcs}(u, v)$ and $(v, u)$ in $D$. These two arcs form a cycle, namely $(u, v)$ in $D$. 


\section{References}

[1] Michael R. Garey, David S. Johnson, Computers and Intractability: A Guide to the Theory of NPcompleteness

[2] The free encyclopedia Wikipedia, https://en.wikipedia.org/wiki/NP-completeness 